\title{
Integrated Analysis of a Competing Endogenous RNA Network Revealing a Prognostic Signature for Cervical Cancer
}

\author{
Leilei Xia ${ }^{1+}$, Han Wang ${ }^{2 \dagger}$, Shengyun Cai ${ }^{1+}$, Xiaoling Su ${ }^{3}$, Jizi Shen ${ }^{1}$, Qi Meng ${ }^{1}$, Yu Chen ${ }^{1}$, \\ $\mathrm{Li} \mathrm{Li}^{1}$, Jiuqiong Yan ${ }^{1}$, Caihong Zhang ${ }^{1}$ and Mingjuan $\mathrm{Xu}^{1 *}$ \\ ${ }^{1}$ Department of Obstetrics and Gynecology, Changhai Hospital, Second Military Medical University, Shanghai, China, \\ ${ }^{2}$ Department of Pathology, Eastern Hepatobiliary Surgery Hospital, Second Military Medical University, Shanghai, China, \\ ${ }^{3}$ Department of Obstetrics and Gynecology, No. 455 Hospital, Shanghai, China
}

Given the high morbidity and the trend of younger individuals being affected observed in cervical cancer, it is important to identify sensitive and effective biomarkers for predicting the survival outcome of patients. Based on data from 307 cervical cancer

OPEN ACCESS

Edited by:

Charles A. Kunos,

National Cancer Institute (NIH),

United States

Reviewed by:

Patrice Mathevet,

Lausanne University Hospital (CHUV),

Switzerland

Salvatore Giovanni Vitale,

Università degli Studi di Messina, Italy

${ }^{*}$ Correspondence:

Mingjuan Xu

13636373419@163.com

tThese authors have contributed equally to this work

Specialty section:

This article was submitted to

Women's Cancer,

a section of the journal

Frontiers in Oncology

Received: 29 June 2018

Accepted: 20 August 2018

Published: 06 September 2018

Citation:

Xia L, Wang H, Cai S, Su X, Shen J, Meng Q, Chen Y, Li L, Yan J, Zhang C and Xu M (2018) Integrated Analysis

of a Competing Endogenous RNA

Network Revealing a Prognostic

Signature for Cervical Cancer.

Front. Oncol. 8:368.

doi: 10.3389/fonc.2018.00368 cases acquired from The Cancer Genome Atlas portal, 1920 differentially expressed mRNAs, 70 microRNAs(miRNAs), and 493 long non-coding(IncRNAs) were screened by comparing cervical cancer tissues with paracancerous tissues. A competing endogenous (ceRNA) network containing 50 IncRNAs, 16 miRNAs, and 81 mRNAs was constructed. Eighteen RNAs, comprising 13 mRNAs, 2 miRNAs, and 3 IncRNAs, were identified as significant prognostic factors by univariate Cox proportional hazards regression. ETS-related gene and fatty acid synthase signatures were discovered using a multivariate Cox regression model built to identify independent prognostic factors in cervical cancer patients. Receiver operating characteristic (ROC) analysis was used to determine the optimal cut-off value for distinguishing the risk level of cervical cancer patients. High-risk patients exhibited a poorer prognosis than low-risk patients did. This study focused on ceRNA networks to provide a novel perspective and insight into cervical cancer and suggested that the identified signature can serve as an independent prognostic biomarker in cervical cancer.

Keywords: cervical cancer, ceRNA network, prognostic factor, FASN, ERG

\section{INTRODUCTION}

Cervical cancer (CC) is one of the leading malignant carcinomas threatening women's health and represents a worldwide concern (1). Since the introduction of the Papanicolaou test and human papilloma (HPV) DNA test, the incidence and mortality of CC has dramatically reduced (2). The long transmit time from cervical intraepithelial lesions to CC provides an opportunity to identify pre-cancerous lesions and enable early treatment. Once the disease develops into CC, the overall survival rate directly depends on stage, with an average survival of $70 \%$ at 5 years (3). However, approximately $11.4 \%$ of women are not screened using the effective screening systems mentioned above (4). Although HPV was identified as a driving factor that can lead to CC, a substantial proportion of CC tissues are HPV-negative (5). Given the high morbidity of this disease and the trend of younger individuals being affected (6), it is important to identify sensitive and effective biomarkers. 
mRNA, microRNA(miRNA), long non-coding(lncRNA), and competing endogenous (ceRNA) represent a new RNA mechanism that regulates gene expression. Assessing miRNA regulatory networks to provide new perspectives for evaluating cancer regulatory networks is complex (7). Using miRNA as a bridge, lncRNA can modulate cell functions by regulating mRNA expression levels.

The Cancer Genome Atlas (TCGA) is a publicly available dataset that provides many types of genomic data (8). This information can be mined to construct a lncRNA-miRNAmRNA ceRNA network in CC. In this study, we obtained RNA-seq profiles from TCGA and performed integrated analysis of differentially expressed RNAs. By using Cox regression analysis, a signature based on ETS-related gene (ERG) and fatty acid synthase (FASN) showed potential as a biomarker with prognostic value in CC.

\section{METHODS AND MATERIALS}

\section{Data Source and Clinical Information}

High-throughput sequencing-counts (HTSeq-counts) and miRNA sequencing profiles were obtained from TCGA data portal, which included 307 CC patients as of March 1, 2018. Three profiles were obtained from normal or adjunct cervical tissues, whereas the other signatures were obtained from CC tissues.

Clinical information from CC patients was also downloaded from TCGA. Of the 307 patients, 254 patients had cervical squamous cell carcinoma and 53 patients had adenocarcinoma carcinoma. The median age of these population was 46 years. According to clinical stage, 188 individuals were stage I-IIa while 109 individuals were stage IIb-IV. According to the status after surgical treatment, 191 patients were tumor free, whereas 74 patients survived with tumors. At the time of the last followup, 247 patients were still alive, whereas 60 had died. Infection with HPV was not consistently reported, with only 15 patients reported to have HPV. Thus, the HPV subgroup was not analyzed.

\footnotetext{
Abbreviations: TCGA, The Cancer Genome Altas; CeRNA, competing endogenous RNA; ERG, ETS Transcription Factor; FASN, Fatty Acid Synthase; ROC, receiver operating characteristic curve; HPV, Human Papillomavirus; HTSeq, high-throughput sequencing; GO, gene ontology; KEGG, Kyoto Encyclopedia of Genes and Genomes; EGFR, Epidermal Growth Factor Receptor; BMI, Body Mass Index; FOXO1, Forkhead Box O1; KLF5, Kruppel Like Factor 5; PRLR, Prolactin Receptor; THBS1, Thrombospondin 1; CCNB1, Cyclin B1; BCL2, B-Cell CLL/Lymphoma 2; IGFBP5, Insulin Like Growth Factor Binding Protein 5; BAK1, BCL2 Antagonist/Killer 1; FGF2, Fibroblast Growth Factor 2; TXNIP, Thioredoxin Interacting Protein; MYB, MYB Proto-Oncogene; AKT3, AKT Serine/Threonine Kinase 3; IDH2, Isocitrate Dehydrogenase [NADP $(+)]$ 2, Mitochondrial; FLI1, Friend Leukemia Virus Integration 1; CDC25A, Cell Division Cycle 25A; SLC43A1, Solute Carrier Family 43 Member 1; MYB, MYB Proto-Oncogene; mTOR, Mechanistic Target of Rapamycin Kinase; HIF1-1 $\alpha$, Hypoxia Inducible Factor 1 Alpha Subunit; BCL2, B-Cell CLL/Lymphoma 2; COX-2, Mitochondrially Encoded Cytochrome C Oxidase II; ETS, erythroblast transformation-specific; TMPRSS2, Transmembrane Protease, Serine 2; FZD4, Frizzled Class Receptor 4; CNS, central nervous system; PPI, Protein protein interactions.
}

\section{Identification of Differentially Expressed mRNAs, miRNAs, and IncRNAs}

All expression profiles were normalized within and among samples. mRNA and lncRNA were classified by their HTSeqcounts based on CRCh38.87 mapping. To identify potential RNAs involved in the development of CC, differentially expressed RNAs between normal or adjunct cervical tissues and CC tissues were analyzed using the edgeR package with a cutoff of false discovery rate-adjusted $p<0.01$ and $|\log \mathrm{FC}| \geq 2$ (9). Because many RNAs exhibit low expression, RNAs with a mean expression of less than 1 were excluded. The heatmap was plotted using the heatmap. 2 function in gplots package.

\section{Construction of the ceRNA Network}

Because mRNA, miRNA, and lncRNA can regulate each other, it is necessary to construct a ceRNA network to provide insight into their interaction mechanisms. Thus, we set miRNA as the central point and differentially expressed RNAs were analyzed based on the following pairings: miRNA-lncRNA and mRNA-miRNA. Briefly, according to the miRcode database (10), differentially expressed lncRNAs were used to pair differentially expressed miRNAs. Next, the paired miRNAs were used to identify target mRNAs according to three databases: miRDB (11), miRTarBase (12), and TargetScan (13). Only mRNAs predicted by all three databases were defined as target mRNAs. Next, the intersection of target mRNAs and differentially expressed mRNAs was selected to construct the ceRNA network along with the miRNAs and lncRNAs mentioned above.

\section{Functional Enrichment Analysis of Gene Ontology and KEGG Pathways}

The intersections of target mRNAs and differentially expressed mRNAs were subjected to functional enrichment analysis, including gene ontology and KEGG pathway analysis. The mRNAs involved in the ceRNA network were divided into two groups: upregulated mRNAs and downregulated mRNAs. The clusterProfiler package was utilized to analyze function enrichment (14). A $p<0.05$ was considered to indicate significant enrichment.

\section{Construction of a Prognostic Signature Based on the ceRNA Network}

Univariate Cox proportional hazards regression analyses were performed based on mRNAs, miRNAs, and lncRNAs in the ceRNA network. Prognosis-related RNAs were defined using the cutoff of $p<0.05$. Next, a multivariate Cox proportional hazards regression model was constructed based on RNAs with a $p<0.01$ to predict the risk score of each patient based on the expression of RNA. The risk score for predicting overall survival was calculated as follows: $\exp _{\mathrm{RNA} 1} * \beta_{\mathrm{RNA1}}+$ $\exp _{\mathrm{RNA} 2} * \beta_{\mathrm{RNA} 2}+\exp _{\mathrm{RNA} 3} * \beta_{\mathrm{RNA} 3}+\ldots \exp _{\mathrm{RNAn}} * \beta_{\mathrm{RNAn}}$, where $\exp _{\mathrm{RNA}}$ is the expression level of RNA and $\beta_{\mathrm{RNA}}$ is the regression coefficient calculated by the multivariate Cox proportional hazards regression model. Patients were divided into a lowrisk group and high-risk group according to the mean risk score. Kaplan-Meier curve analysis was conducted to compare 
the survival times of the low-risk group and high-risk group. $P<0.05$ was considered statistically significant. Chi-square test was utilized to correlate risk level with clinical parameters including age, race, body mass index (BMI), menopause status, histological type, tumor grade, clinical stage, neoplasm status, and vital status. Receiver operating characteristic (ROC) curve analysis using 3 years as the predicted time was also performed to estimate the predictive value of the outcome. The area under the ROC curve along with sensitive and specificity were used as evaluation indices of the prediction value.

A Cox proportional hazards regression model was further used to better evaluate the effects of clinical characteristics and risk score on the overall survival of CC patients.

\section{Protein-Protein Interaction Network Construction}

The protein-protein interaction network was constructed using the STRING website (15), which is a tool for analyzing the relationship between proteins and scoring these relationships based on experimental data and forecast data. The interaction network was visualized using Cytoscape software.

\section{Statistical Analysis}

In the analysis of differently expressed genes, significance was tested using the optimal discovery procedure and generalized likelihood ratio tests. $p<0.01$ and $|\log \mathrm{FC}| \geq 2$ were considered statistically significant.

Enumeration data were described by sample size and constituent ratio and chi-square test was utilized correlate the risk level with clinical parameters. A univariate Cox proportional hazards model was used to assess the risk factors associated with the outcomes of CC patients, and then clinical parameters with $p<0.05$ were entered in multivariate Cox proportional hazards model to evaluate prognostic factors. Kaplan-Meier plot analysis was used to determine the overall survival rate and the Log-rank test was utilized to analyze the differences between groups. The ROC curve was applied to calculate the sensitivity and specificity of risk score. $P<0.05$ was considered statistically significant.

\section{RESULTS}

\section{Identification of Differentially Expressed mRNAs, miRNAs, and IncRNAs}

A total of 19676 mRNAs, 1881 miRNAs, and 14487 lncRNAs were extracted from TCGA dataset. Differentially expressed RNAs were analyzed using the edgeR package following extraction of the expression matrix from 307 CC cases. Using $p<0.05$ and $|\log F C| \geq 2$ as the cut-off criteria, 1920 differentially expressed mRNAs (712 upregulated and 1208 downregulated), 70 differentially expressed miRNAs (33 upregulated and 37 downregulated), and 493 differentially expressed lncRNAs (128 upregulated and 365 downregulated) were identified by comparing CC and adjacent normal cervical tissues (Supplement Figure 1).

\section{Construction of the ceRNA Network Based on Predicted miRNA Targets}

Numerous studies have suggested that lncRNAs interact with miRNA response elements to act as miRNA sponges $(16,17)$. First, the target regulation network of IncRNA-miRNA was constructed. Using miRcode to screen for potential miRNA response elements of lncRNA, 242 potential lncRNA-miRNA pairs, including $50 \operatorname{lncRNAs}$ and 18 miRNAs, were formed (Supplement Table 1). Next, the miRNA-mRNA target regulation network was further assessed using miRDB, TargetScan, and miRTarBase. In total, 98 miRNA-mRNA pairs were identified, including $81 \mathrm{mRNAs}$ and 16 miRNAs (Supplement Table 2). The lncRNA-miRNA-mRNA network was then constructed using Cytoscape software based on the results above and contained 50 lncRNAs, 16 miRNAs, and 81 mRNAs (Figure 1).

\section{Enrichment Analysis of Gene Ontology and KEGG Pathways}

In total, 81 mRNAs were involved in the ceRNA network: 31 upregulated mRNAs and 50 downregulated mRNAs. ClusterProfiler package was utilized to compare gene clusters based on their enriched biological processes with a cutoff of $p<0.05$. In GO functional enrichment analysis, the upregulated mRNAs that were significantly enriched included cyclin-dependent protein serine/threonine kinase regulator activity, histone kinase activity, protein kinase regulator activity, kinase regulator activity, and chaperone binding. The downregulated mRNAs that were significantly enriched included transforming growth factor beta binding, growth factor binding, transcriptional repressor activity, RNA polymerase II transcription regulatory region sequence-specific binding, transcription corepressor activity, transcription factor activity, and RNA polymerase II core promoter proximal region sequence-specific binding (Supplement Table 3). In the KEGG analysis, the upregulated mRNAs were mainly enriched in p53 signaling pathway, cell cycle, cellular senescence, microRNAs in cancer, and small cell lung cancer. Downregulated mRNAs were mainly enriched in prostate cancer, transcriptional misregulation in cancer, melanoma, epidermal growth factor receptor (EGFR) tyrosine kinase inhibitor resistance, and microRNAs in cancer (Supplement Table 4) (Figure 2).

\section{Construction of a Prognostic Signature Based on the ceRNA Network}

We further performed survival and prognostic analyses of the 50 lncRNAs, 16 miRNAs, and 81 mRNAs in the ceRNA network. Univariate Cox proportional hazards regression revealed that 18 RNAs, comprised of 13 mRNAs, 2 miRNAs, and 3 lncRNAs, were identified as significant prognostic factors based on the cutoff of a $p<0.05$ (Supplement Table 5). Multivariate Cox proportional hazards regression analysis revealed that two RNAs exhibited a significant prognostic value: ERG and FASN (Supplement Table 6) (Figure 3). The risk assessment score for predicting overall survival was calculated as follows: Risk score $=$ $\exp _{\mathrm{ERG}} * 0.359+\exp _{\mathrm{FASN}} * 0.345$. Based on the risk score, cervical 


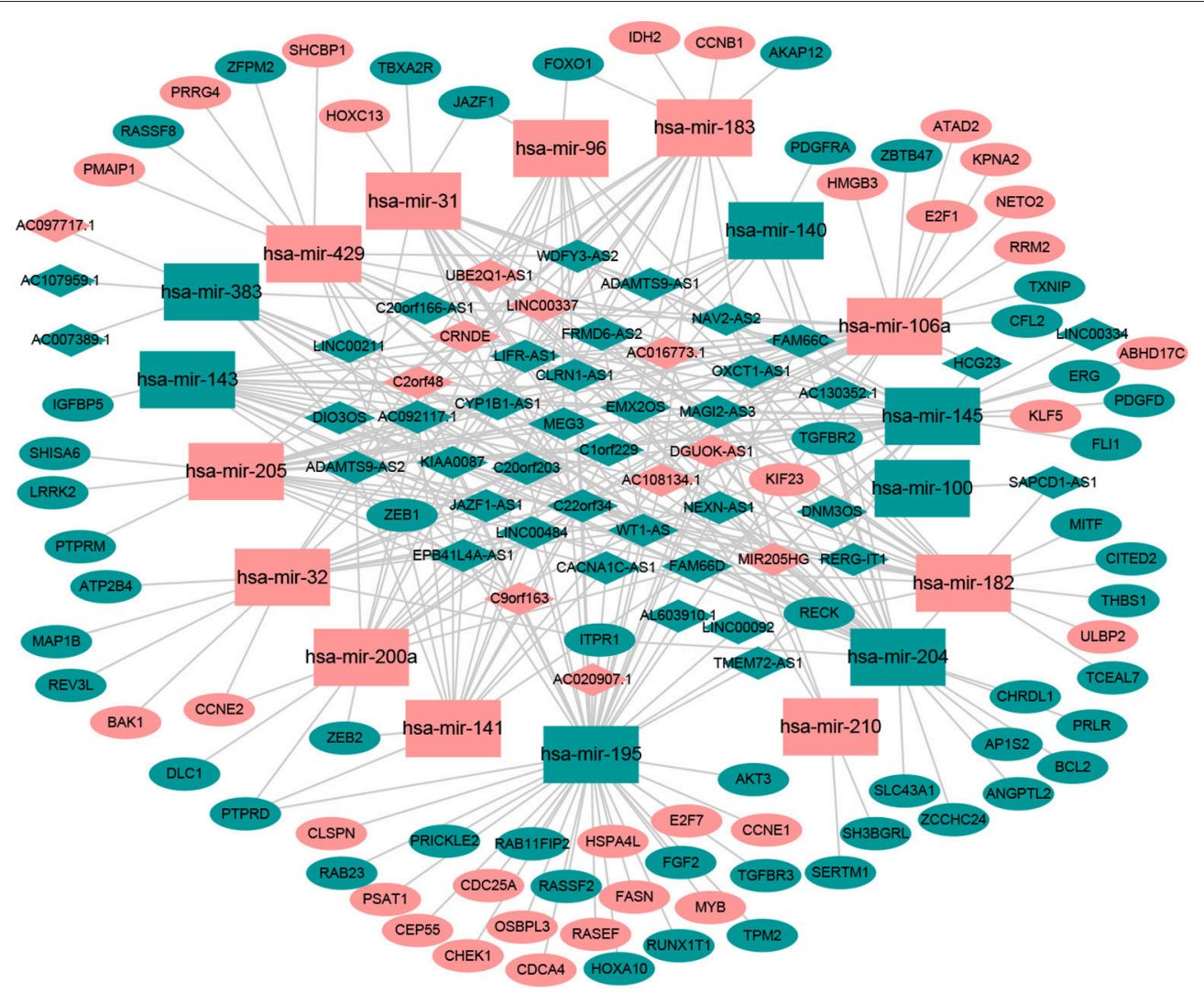

FIGURE 1 | The ceRNA network of IncRNA-miRNA-mRNA. Green diamonds represent downregulated IncRNA, red diamonds indicate upregulated IncRNA, green rectangles indicate downregulated miRNA, red rectangles indicate upregulated miRNA, green ellipses indicate downregulated mRNA, and red ellipses indicate upregulated mRNA.

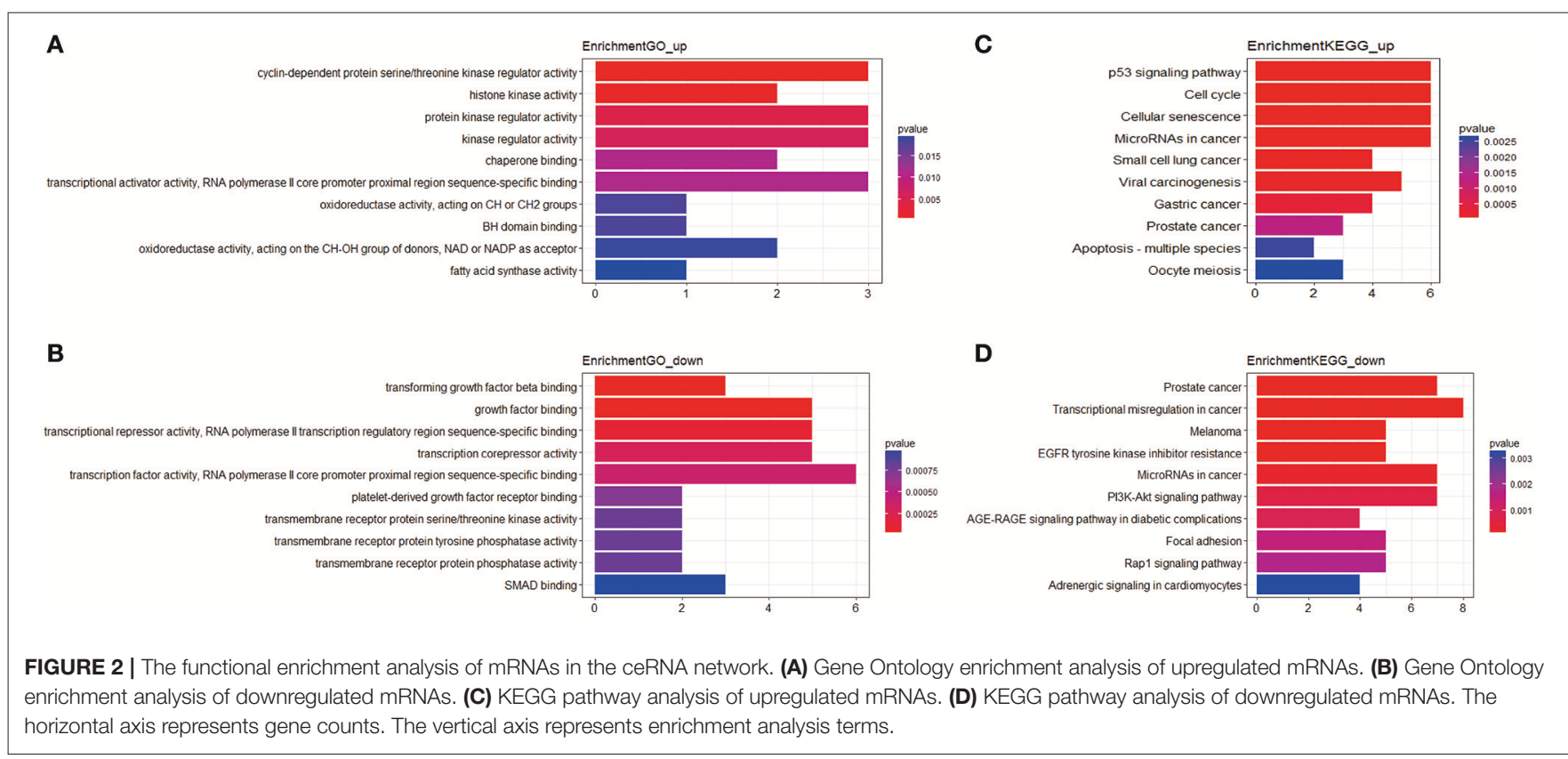


A

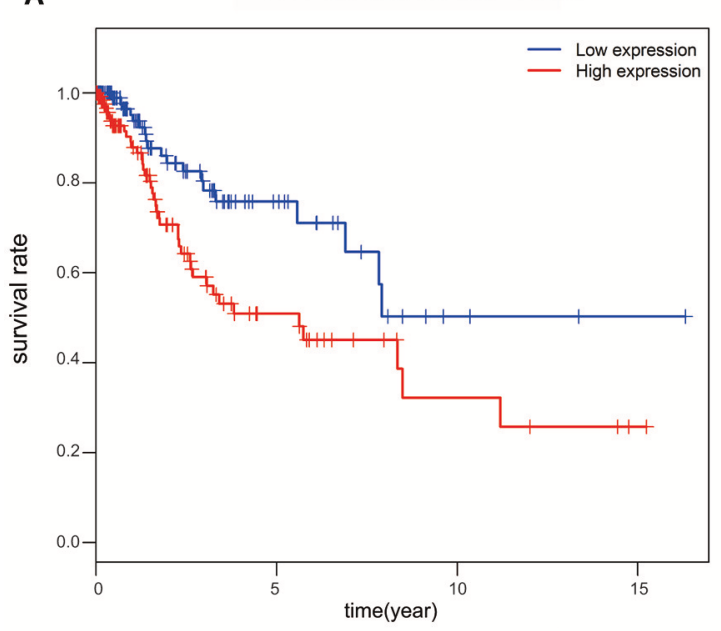

B

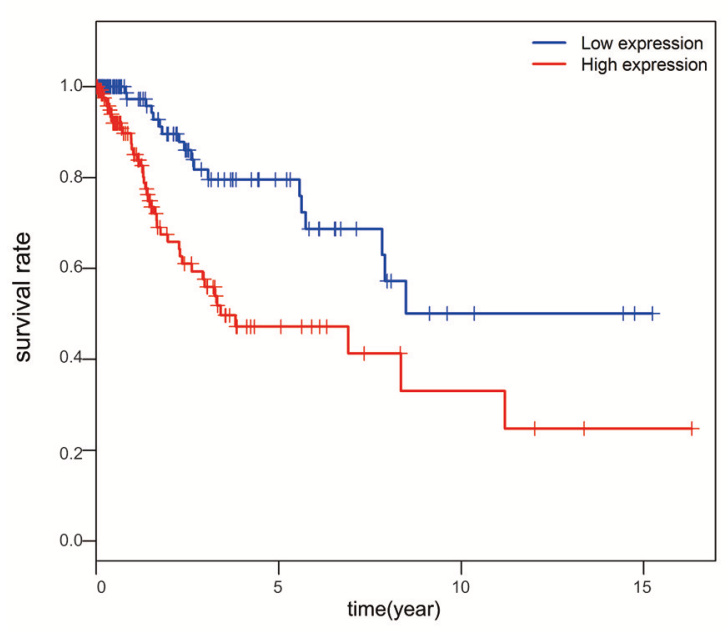

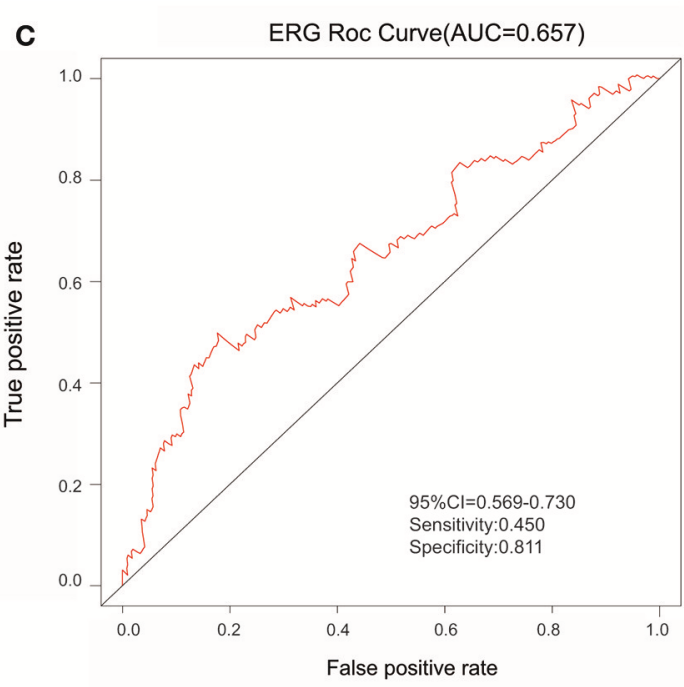

D

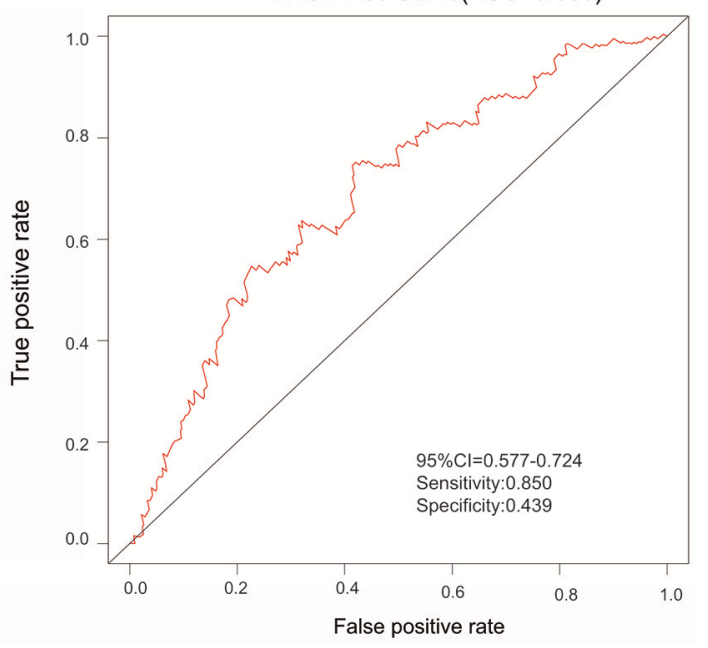

FIGURE 3 | The predictive value of ERG and FASN on outcome. (A) The Kaplan-Meier survival analysis of ERG expression in overall survival. (B) The Kaplan-Meier survival analysis of FASN expression in overall survival. (C) ROC cure for predicting 3-year survival based on ERG expression levels. (D) ROC curve for predicting 3-year survival based on FASN expression level.

cancer patients were divided into two group: low-risk and highrisk. Kaplan-Meier survival analysis indicated that the mean survival time was 2154 days for the high-risk group and 4166 days for the low-risk group $(p<0.001)$. In ROC curve analysis, the 3 year survival of the area under the curve was $0.718(p<0.001$, 95\% confidence interval of hazard ratio: $0.613-0.766$, sensitivity: 0.733, specificity: 0.582) (Figure 4).

The clinical parameters along with risk level are shown in Table 1. In the Chi-square test, as shown in Table 2, the risk level was significantly related to BMI $(p=0.006)$, histological type $(p<0.001)$, clinical stage $(p=0.001)$, and vital status $(p<0.001)$. No dramatic correlation between the risk level and other clinical parameters such as age, race, or neoplasm status were found.

To better evaluate the prognostic value of the risk score, clinical characteristics, including race, age, tumor grade, clinical stage, pathological type, type of neoplasm, BMI, and menopause status, were examined in survival analysis. Univariate analysis of clinicopathological parameters revealed that survival time was significantly associated with clinical stage $(p=0.02)$, type of neoplasm $(p<0.001)$, BMI $(p=0.04)$, and risk score $(p<0.001)$. However, no significant differences were found between the survival time and race, age, tumor grade, pathological type or menopause status (Figure 5). Unexpectedly, multivariate Cox proportional hazards regression analysis indicated that type of neoplasm $(p<0.001)$ and risk level $(p=0.04)$ were significantly independent predictive factors of poorer prognosis for CC but not clinical stage $(p>0.05)$ (Figure 6).

\section{Protein-Protein Network Analysis}

Based on the differentially expressed mRNAs, the protein-protein network was constructed using FASN and ERG as the center. We 

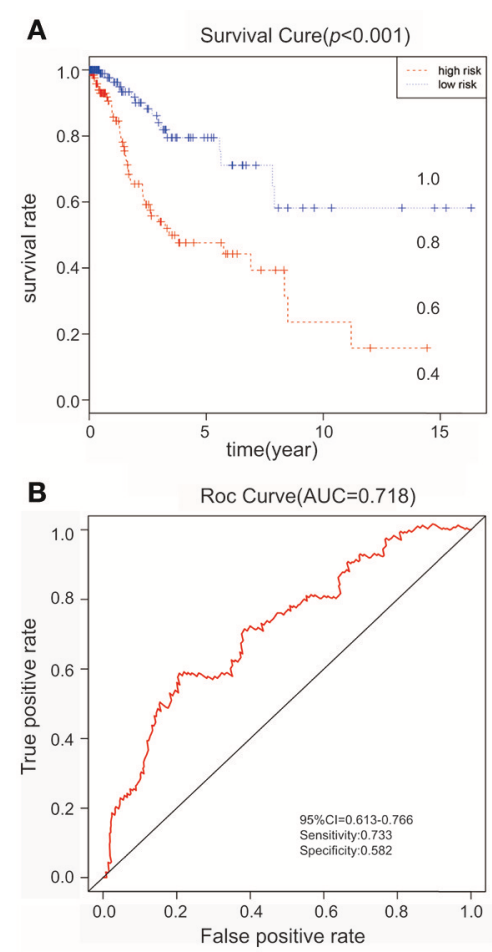

c

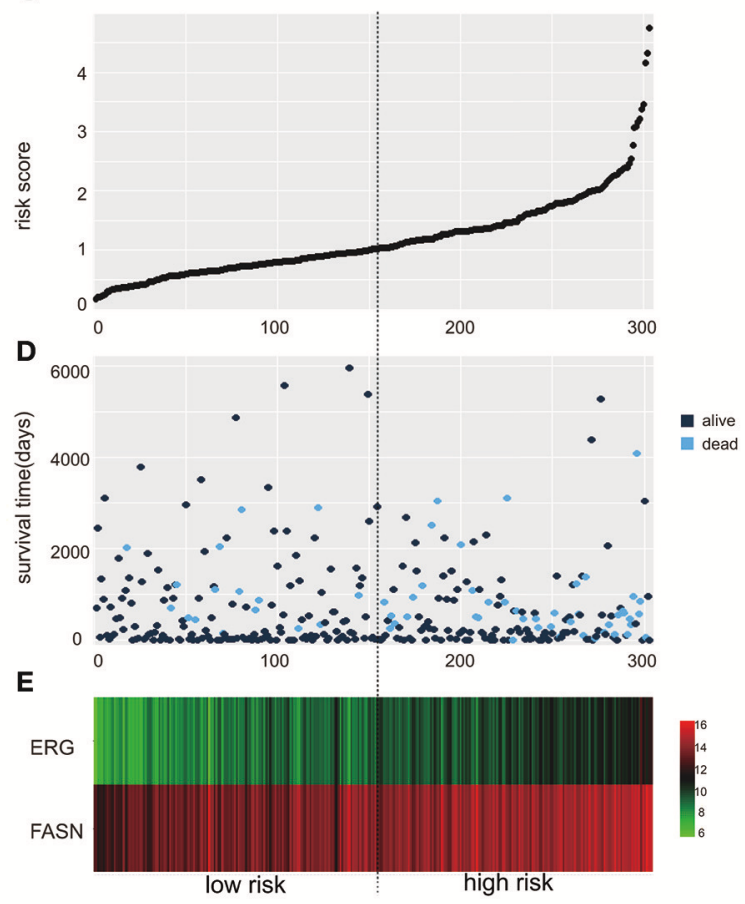

FIGURE 4 | The predictive value of the risk score calculated by ERG and FASN on outcome. (A) Kaplan-Meier survival analysis of the risk score for overall survival. (B) ROC curve for predicting 3-year survival based on risk score. (C) Risk score of each individual. (D) Survival status and survival time of each individual. (E) Heatmap of ERG and FASN in high-risk and low-risk groups.

found that FASN was related to FOXO1, KLF5, PRLR, THBS1, CCNB1, BCL2, IGFBP5, BAK1, FGF2, TXNIP, MYB, AKT3, and IDH2, whereas ERG was closely related to FLI1, CDC25A, SLC43A1, and MYB (Figure 7).

\section{DISCUSSION}

Screening systems such as the Pap and HPV DNA test dramatically reduced the incidence and morality of CC, but its high sensitivity and low specificity contribute to colposcopy in numerous women with doubtful lesions. Increasing studies have performed to identify biomarkers to distinguish disease stages and some progress has been made. P16ink4a, p16, E-cadherin, Ki67, pRb, and p53 are markers that can discriminate $\mathrm{CC}$ from intraepithelial lesions, while CEA, SCC, and CD44 are used to detect invasive cancer (3). Among them, P16ink4a is directly linked to HPV oncogenic action, and is overexpressed in nearly all cases of high-grade squamous intraepithelial lesion but rarely in low-grade squamous intraepithelial lesion (18). P16ink4a and Ki67 can improve the sensitivity and specificity of the equivocal atypical squamous cells of undetermined significance or low-grade squamous intraepithelial lesion Pap test results, reducing the requirement for colposcopy.

In this study, differentially expressed RNAs, including mRNA, miRNA, and lncRNA, were identified by comparing adjunct or normal cervical tissues with CC tissues. Using miRNA as a bridge, paired lncRNA-miRNA-mRNAs were screened to construct a ceRNA network. To explore the effect of the ceRNA network on survival status, univariate and multivariate Cox proportional hazard regression analyses were conducted to successively analyze the prognostic value of differentially expressed RNAs. According to the expression level of prognostic genes, CC patients were divided into low-risk and high-risk groups. Next, clinical characteristics and risk scores were analyzed. TNM stage, BMI, type of neoplasm, and risk score were identified as prognostic factors of CC patients.

Compared to mRNA, IncRNA was recently discovered. Abundant evidence indicates that lncRNAs interact with the miRNA response element and function as miRNA sponges. Using miRNA as a bridge, ceRNA networks involving mRNA and IncRNA have been constructed, providing insight into CC. Previous studies revealed interactions between miRNA and mRNA. Functional analysis of differentially expressed miRNA targets revealed numerous cancer-associated pathways, cell growth and death-related pathways, including apoptosis, cell cycle, p53 signaling pathway and other signal transduction pathways, such as ErbB, MAPK, mTOR, Notch, TGF- $\beta$, and Wnt. Particularly, based on bioinformatics analysis of datasets including GEO and TCGA, key genes have been identified and found to be involved in cell proliferation including TP53, MYC, CCND1, CDKN2A, JUN, FOS, which are targets of differentially expressed miRNAs in CC. These transcription regulators may 
TABLE 1 | Clinical parameters of cervical cancer patients.

\begin{tabular}{|c|c|c|c|}
\hline Subgroup & Frequency & Percent & Valid percent \\
\hline \multicolumn{4}{|l|}{ Age } \\
\hline$<60$ & 238 & 78.6 & 78.6 \\
\hline$>=60$ & 65 & 21.4 & 21.4 \\
\hline \multicolumn{4}{|l|}{ Race } \\
\hline White & 209 & 68.8 & 91.3 \\
\hline Asian & 20 & 6.6 & 8.7 \\
\hline \multicolumn{4}{|l|}{ BMl } \\
\hline$<25$ & 98 & 32.2 & 37.8 \\
\hline$>=25$ & 161 & 53 & 62.2 \\
\hline \multicolumn{4}{|l|}{ Menopause } \\
\hline Premenopausal & 124 & 40.8 & 53.7 \\
\hline Perimenopause/postmenopausal & 107 & 35.2 & 46.3 \\
\hline \multicolumn{4}{|l|}{ Histological type } \\
\hline Squamous cellcarcinoma & 252 & 82.9 & 82.9 \\
\hline $\begin{array}{l}\text { Adenocarcinoma/adenosquamous } \\
\text { carcinoma }\end{array}$ & 52 & 17.1 & 17.1 \\
\hline \multicolumn{4}{|l|}{ Grade } \\
\hline $\mathrm{G} 1+\mathrm{G} 2$ & 153 & 50.3 & 56.3 \\
\hline G3 & 119 & 39.1 & 43.8 \\
\hline \multicolumn{4}{|l|}{ Clinical stage } \\
\hline I-IIA2 & 188 & 61.8 & 63.3 \\
\hline IIB-IV & 109 & 35.9 & 36.7 \\
\hline \multicolumn{4}{|l|}{ Neoplasm status } \\
\hline Tumor free & 188 & 61.8 & 71.8 \\
\hline With tumor & 74 & 24.3 & 28.2 \\
\hline \multicolumn{4}{|l|}{ Vital status } \\
\hline Alive & 244 & 80.3 & 80.3 \\
\hline Dead & 60 & 19.7 & 19.7 \\
\hline \multicolumn{4}{|l|}{ Risk level } \\
\hline Low & 152 & 50 & 50 \\
\hline High & 152 & 50 & 50 \\
\hline
\end{tabular}

form feedback loops with miRNAs to modulate cell apoptosis and migration in CC. A pioneer study based on the interaction of mRNA and miRNA indicated that CHEK1, SOX17, CDKN2A, and CEP55, which are targets of star miRNAs in CC including miR-424-5p, miR-200a-5p, miR-9-5p, and miR-133b, play a vital role in the development of CC (19). However, the combination of IncRNA, miRNA and mRNA expression profile has not widely examined in CC. In this study of ceRNA, 50 lncRNAs, 16 miRNAs, and 81 mRNAs were found to be involved in cervical carcinogenesis.

miRNA are short regulatory RNAs that modulate gene expression levels by partial base pairing with the $3^{\prime}$ untranslated region of their target mRNAs. miRNAs show partial nucleotide sequence paring between miRNAs and their mRNA targets, leading to promiscuous interactions: one miRNA may interact with one or more mRNAs and one mRNA may be targeted by multiple miRNAs. miRNAs play vital roles in maintaining physiological function, including development, metabolism, senile, and apoptosis. Dysregulation of miRNAs can result in various diseases.
TABLE 2 | Relationship between risk level and clinical parameters.

\begin{tabular}{|c|c|c|c|c|}
\hline Subgroup & High-risk & Low-risk & Total & $P$-value \\
\hline Age & & & & 0.484 \\
\hline$<60$ & 122 & 117 & 239 & \\
\hline$>=60$ & 30 & 35 & 65 & \\
\hline Race & & & & 0.684 \\
\hline White & 105 & 104 & 209 & \\
\hline Asian & 11 & 9 & 20 & \\
\hline $\mathrm{BMl}$ & & & & $0.006^{\star}$ \\
\hline$<25$ & 58 & 40 & 98 & \\
\hline$>=25$ & 67 & 94 & 161 & \\
\hline Menopause & & & & 0.315 \\
\hline Premenopausal & 65 & 59 & 124 & \\
\hline $\begin{array}{l}\text { Perimenopause/ } \\
\text { postmenopausal }\end{array}$ & 49 & 58 & 107 & \\
\hline Histological type & & & & $<0.001^{*}$ \\
\hline Squamous cellcarcinoma & 139 & 113 & 252 & \\
\hline $\begin{array}{l}\text { Adenocarcinoma/ } \\
\text { adenosquamous } \\
\text { carcinoma }\end{array}$ & 13 & 39 & 52 & \\
\hline Grade & & & & 0.783 \\
\hline $\mathrm{G} 1+\mathrm{G} 2$ & 72 & 81 & 153 & \\
\hline G3 & 58 & 61 & 119 & \\
\hline Clinical stage & & & & $0.001^{*}$ \\
\hline I-IIA2 & 80 & 108 & 188 & \\
\hline IIB-IV & 68 & 41 & 109 & \\
\hline Neoplasm statue & & & & 0.17 \\
\hline Tumor free & 89 & 99 & 188 & \\
\hline With tumor & 42 & 32 & 74 & \\
\hline Vital status & & & & $<0.001^{*}$ \\
\hline Alive & 108 & 136 & 244 & \\
\hline Dead & 44 & 16 & 60 & \\
\hline
\end{tabular}

${ }^{*} p<0.05$.

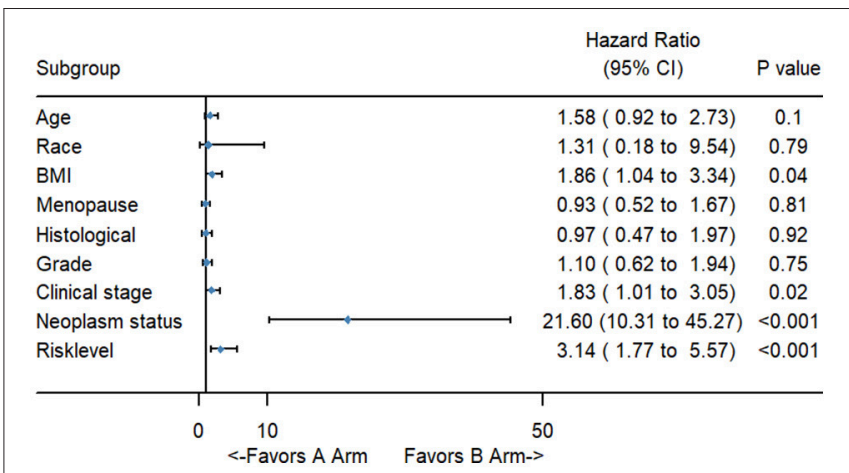

FIGURE 5 | The forest map of univariate logistic regression analysis. The line provided an imaginal of $95 \% \mathrm{Cl}$, location of diamond on the line represented the odds ratio.

In the progression of $\mathrm{CC}$, miRNAs were analyzed according to the severity of disease including CIN1, CIN2, CIN3, and CC. Few changes were observed in the miRNA expression in CIN1 compared to in normal cervical tissue. As the disease progressed, increasing number of miRNAs was found to be greatly altered (20). 
In preeclampsia (PE), the perturbation of angiogenesis is recognized as a vital pathogenic factor. Various studies demonstrated that miR-16, miR-26b, miR-29b, miR-335, miR222, miR-181a, and miR-195, which are associated with vascular endothelial growth factor, were aberrantly upregulated in PE (21). Among them, miR-181a was over-expressed in both the placenta and maternal sera. Additionally, miR-181, acting as modulator of T-cell sensitivity and is associated with tolerance induction at the maternal-fetal interface, was disturbed during PE (22).

Placental vascular alterations due to angiogenic imbalance occurred in PE as well as fetal growth restriction (FGR). FGR is the termination or decrease of the fetus determined by generic factors during pregnancy. Accumulating evidence demonstrated that the dysregulation of miRNAs is involved in FGR. In placental tissue, miR-21, miR-499a-5p, miR-1-3p, miR-424, miR141, and miR-519a are overexpressed in FGR while miR-194 are downregulated (23). In maternal circulation, miR-100-5p, miR125-5p, and miR-199a-5p are downregulated in FGR as well as preeclampsia and gestational hypertension (24). In vitro and in vivo experiments showed that, miR-141-3p and miR-200A-3p are vital for placental development in mouse by regulating the level of insulin like growth factor 2 (25).

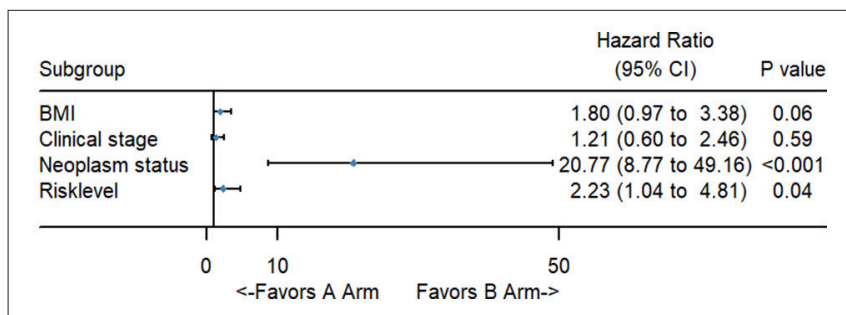

FIGURE 6 | The forest map of multivariate logistic regression analysis. The line provided an imaginal of $95 \% \mathrm{Cl}$, location of diamond on the line represented the odds ratio.
The survival outcome of CC is mainly predicted by two elements, TNM stage and type of neoplasm; however, these indices are not quantifiable. Some studies suggested that hypoxiainducible factor- $1 \alpha$ can serve as an independent poor survival marker of CC, as it is involved in numerous pathways, including the PI3K/AKT and mTOR pathways (26). Additionally, hypoxiainducible factor- $1 \alpha$ may be induced by HPV to promote tumor angiogenesis (27). BCL-2, a key protein in the process of transmitting apoptosis signals, is negatively correlated with disease-free survival in CC and can function as an independent survival factor (28). Cyclooxygenase-2, a cyclooxygenase subtype, can inhibit the apoptosis signal, increase vascularization, and activate the PI3K/AKT pathway to promote chemoresistance (29). Additionally, EGFR was upregulated with the degree of malignancy in CC. EGFR exhibits a positive correlation with lymph node metastasis (30). However, an efficient and sensitive marker for predicting the outcome of CC is lacking. In this study, we identified a signature based on ERG and FASN to predict the risk score of each patient and evaluate the outcome in combination with information regarding TNM stage and type of neoplasm in cervical cancer patients.

ERG is a member of the erythroblast transformation-specific family that regulates embryonic development, cell proliferation, apoptosis, and angiogenesis. It is indispensable for inducing vascular cell remodeling (31). Under normal circumstances, ERG is highly expressed in the embryonic mesoderm and endothelium. During vascular development, its expression decreases, but the pluripotency of hematopoietic stem cells continues to be regulated (32). An increasing number of studies has indicated that ERG functions in malignant cancers. In acute myeloid leukemia, ERG expression is high and predicts adverse clinical outcome (33). Several studies demonstrated that ERG is highly and consistently expressed in many prostate cancer patients because of gene fusion with TMPRSS2, an androgen-dependent gene (34). ERG serves as an actuation factor in the development of prostate cancer. ERG overexpression is

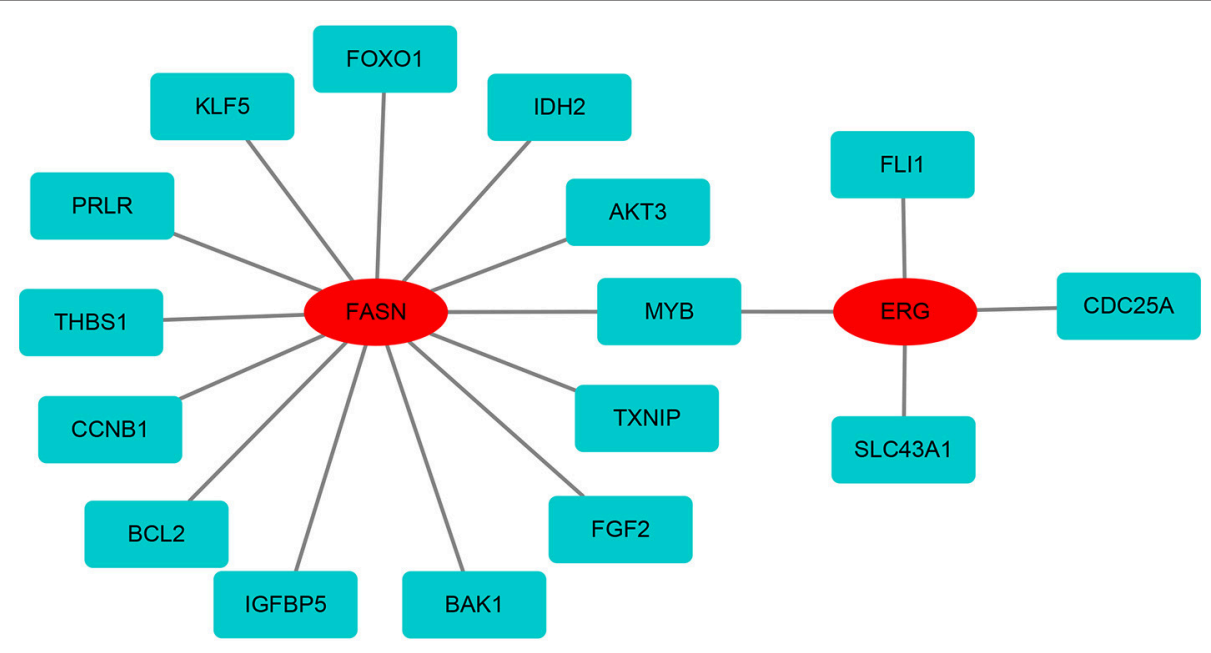

FIGURE 7 | The protein-protein network of FASN and ERG with differentially expressed mRNAs. FASN and ERG were surrounded by differentially expressed mRNAs based on the STRING database. 
associated with advanced TNM stage, high Gleason score, and poor survival outcome (35). ERG overexpression influences the epithelial-mesenchymal transition by upregulating FZD4 and downregulating E-cadherin (36). Additionally, NOTCH and Wnt signaling are activated by high levels of ERG to induce cell migration and invasion. ERG is upregulated in central nervous system tumors, including glioblastomas and hemangioblastomas, and may function as a specific marker in these tumors (37).

FASN encodes an enzyme that catalyzes the conversion of acetyl-CoA and malonyl-CoA into long-chain saturated fatty acids. FASN overexpression is associated with malignant tumors, including breast cancer (38), prostate cancer (39), colorectal cancer (40), gastric cancer (41), and lung cancer (42), as well as poor prognosis. Loss of FASN can reduce the energy metabolism, proliferation, and migration of cancer cells. FASN also inhibits the expression of HER2 and may function as a potential therapeutic target in estrogen receptor- and progesterone receptor-positive endometrial cancers (43).

Few studies have focused on the role and mechanism of ERG and FASN in CC. In our protein-protein interaction network analysis, we found that FASN and ERG proteins interact with other proteins, including FOXO1, KLF5, THBS1, CCNB1, BCL2, AKT3, and CDC25A, to play a crucial role in CC. We identified a signature based on ERG and FASN as a biomarker in CC. This signature may serve as a therapeutic target for precision medicine in CC. This study describes a new method for further investigating the pathogenesis of CC. Further studies are required to explore the biological functions and underlying molecular mechanisms of ERG and FASN in cervical cancer.

\section{CONCLUSION}

This study focused on ceRNA networks to provide a novel perspective and insight into CC and suggested that the signature based on ERG and FASN can serve as an independent prognostic biomarker in CC.

\section{ETHICS STATEMENT}

High-throughput sequencing-counts (HTSeq-counts) and miRNA sequencing profiles were obtained from the TCGA data portal, which is a publicly available dataset for which no ethics approval is needed.

\section{AUTHOR CONTRIBUTIONS}

LX constructed the ceRNA network using R software. HW analyzed the data using SPSS. SC download data from TCGA. $\mathrm{XS}$ used photoshop and illustration software. JS organized the data. QM performed GO and KEGG analysis. YC performed PPI network analysis. LL wrote the manuscript. JY performed survival analysis. CZ polished the language. MX provided guidance.

\section{FUNDING}

This work was supported by National Key Research and Development Program of China (NO: 2016YFC1303100).

\section{SUPPLEMENTARY MATERIAL}

The Supplementary Material for this article can be found online at: https://www.frontiersin.org/articles/10.3389/fonc. 2018.00368/full\#supplementary-material

Supplement Figure 1 | Heatmap of differentially expressed RNAs. (A) The heatmap of 200 randomly selected differentially expressed mRNAs. (B) The heatmap of 70 differentially expressed miRNAs. (C) The heatmap of 200 randomly selected differentially expressed IncRNAs. Red indicates a high level of RNA expression, whereas green indicates low expression.

Supplement Table 1 | Paired IncRNA and miRNA. 50 differently expressed IncRNAs and 18 differently expressed miRNAs paired according to the miRcode database.

Supplement Table 2 | Paired miRNA and mRNA. 98 differently expressed miRNA-mRNA pairs were identified including 81 mRNAs and 16miRNAs based on the three databases: miRDB, miRTarBase, and TargetScan. Only the mRNAs predicted by all the above three databases defined as the target mRNAs.

Supplement Table 3 | Gene ontology function enrichment analysis.

Supplement Table 4 | KEGG pathway analysis.

Supplement Table 5 | The prognostic differently expressed RNAs involved in ceRNA network by univariate cox regression analysis.

Supplement Table 6 | The prognostic differently expressed RNAs involved in ceRNA network by multivariate cox regression analysis. ${ }^{*} P<0.05$.

\section{REFERENCES}

1. Waggoner SE. Cervical cancer. Lancet (2003) 361:2217-25. doi: 10.1016/S0140-6736(03)13778-6

2. Denny L. Cervical cancer: prevention and treatment. Discov Med. (2012) 14:125-31.

3. Valenti G, Vitale SG, Tropea A, Biondi A, Laganà AS. Tumor markers of uterine cervical cancer: a new scenario to guide surgical practice? Updates Surg. (2017) 69:441-9. doi: 10.1007/s13304-017-0491-3

4. Benard VB, Thomas CC, King J, Massetti GM, Doria-Rose VP, Saraiya M, et al. Vital signs: cervical cancer incidence, mortality, and screening - United States, 2007-2012. MMWR Morb Mortal Wkly Rep. (2014) 63:1004-9.

5. Eileen M. Burd. Human papillomavirus and cervical cancer. Clin Microbiol Rev. (2003) 16:1-17. doi: 10.1128/CMR.16.1.1-17.2003
6. Motoki Y, Mizushima S, Taguri M, Takahashi K, Asano R, Kato H, et al. Increasing trends in cervical cancer mortality among young Japanese women below the age of 50 years: an analysis using the Kanagawa populationbased Cancer Registry, 1975-2012. Cancer Epidemiol. (2015) 39:700-6. doi: 10.1016/j.canep.2015.08.001

7. Salmena L, Poliseno L, Tay Y, Kats L, Pandolfi PP. A ceRNA hypothesis: the rosetta stone of a hidden rna language?.Cell (2011) 146:353-8. doi: 10.1016/j.cell.2011.07.014

8. Tomczak K, Czerwinska P, Wiznerowicz M. The cancer genome atlas (TCGA): an immeasurable source of knowledge. Contemp Oncol (Pozn). (2015) 19:A68-77. doi: 10.5114/wo.2014.47136

9. Robinson MD, McCarthy DJ, Smyth GK. edgeR: a bioconductor package for differential expression analysis of digital gene expression data. Bioinformatics (2010) 26:139-40. doi: 10.1093/bioinformatics/btp616 
10. Jeggari A, Marks DS, Larsson E. miRcode: a map of putative microRNA target sites in the long non-coding transcriptome. Bioinformatics (2012) 28:2062-3. doi: 10.1093/bioinformatics/bts344

11. Wong N, Wang X. miRDB: an online resource for microRNA target prediction and functional annotations. Nucleic Acids Res. (2015) 43(Database issue):D146-52. doi: 10.1093/nar/gku1104

12. Chou CH, Chang NW, Shrestha S, Hsu SD, Lin YL, Lee WH, et al. miRTarBase 2016: updates to the experimentally validated miRNAtarget interactions database. Nucleic Acids Res. (2016) 44(D1):D239-47. doi: $10.1093 / \mathrm{nar} / \mathrm{gkv} 1258$

13. Agarwal V, Bell GW, Nam JW, Bartel DP. Predicting effective microRNA target sites in mammalian mRNAs. Elife (2015) 4:e05005. doi: 10.7554/eLife.05005

14. Yu G, Wang LG, Han Y, He QY. clusterProfiler: an R package for comparing biological themes among gene clusters. OMICS (2012) 16:284-7. doi: 10.1089/omi.2011.0118

15. Szklarczyk D, Morris JH, Cook H, Kuhn M, Wyder S, Simonovic M, et al. The STRING database in 2017: quality-controlled protein-protein association networks, made broadly accessible. Nucleic Acids Res. (2017) 45(D1):D362-8. doi: 10.1093/nar/gkw937

16. Xia $\mathrm{T}$, Liao Q, Jiang X, Shao $\mathrm{Y}$, Xiao B, Xi Y, et al. Long noncoding RNA associated-competing endogenous RNAs in gastric cancer. Sci Rep. (2014) 4:6088. doi: 10.1038/srep06088

17. Tan JY, Sirey T, Honti F, Graham B, Piovesan A, Merkenschlager M, et al. Extensive microRNA-mediated crosstalk between lncRNAs and mRNAs in mouse embryonic stem cells. Genome Res. (2015) 25:655-66. doi: $10.1101 /$ gr.181974.114

18. Zhang HS, Postigo AA, Dean DC. Active transcriptional repression by the Rb-E2F complex mediates G1 arrest triggered by p16INK4a, TGFbeta, and contact inhibition. Cell (1999) 97:53-61.

19. Zhao L, Zhang Z, Lou H, Liang J, Yan X, Li W, et al. Exploration of the molecular mechanisms of cervical cancer based on mRNA expression profiles and predicted microRNA interactions. Oncol Lett. (2018) 15:8965-72. doi: 10.3892/ol.2018.8494

20. He Y, Lin J, Ding Y, Liu G, Luo Y, Huang M, et al. A systematic study on dysregulated microRNAs in cervical cancer development. Int J Cancer (2016) 138:1312-27. doi: $10.1002 / \mathrm{ijc} .29618$

21. Laganà AS, Vitale SG, Sapia F, Valenti G, Corrado F, Padula F, et al. miRNA expression for early diagnosis of preeclampsia onset: hope or hype? J Matern Fetal Neonatal Med. (2018) 31:817-21. doi: 10.1080/14767058.2017.1296426

22. Henao-Mejia J, Williams A, Goff LA, Staron M, Licona-Limón P, Kaech SM, et al. The microRNA miR-181 is a critical cellular metabolic rheostat essential for NKT cell ontogenesis and lymphocyte development and homeostasis. Immunity (2013) 38:984-97. doi: 10.1016/j.immuni.2013.02.021

23. Chiofalo B, Laganà AS, Vaiarelli A, La Rosa VL, Rossetti D, Palmara V, et al. Do miRNAs play a role in fetal growth restriction? a fresh look to a busy corner. Biomed Res Int. (2017) 2017:6073167. doi: 10.1155/2017/6073167

24. Hromadnikova I, Kotlabova K, Hympanova L, Krofta L. Gestational hypertension, preeclampsia and intrauterine growth restriction induce dysregulation of cardiovascular and cerebrovascular disease associated microRNAs in maternal whole peripheral blood. Thromb Res. (2016) 137:12640. doi: 10.1016/j.thromres.2015.11.032

25. Saha S, Choudhury J, Ain R. MicroRNA-141-3p and miR-200a-3p regulate insulin-like growth factor 2 during mouse placental development. Mol Cell Endocrinol. (2015) 414:186-93. doi: 10.1016/j.mce.2015.07.030

26. Huang M, Chen Q, Xiao J, Yao T, Bian L, Liu C, et al. Overexpression of hypoxia-inducible factor- $1 \alpha$ is a predictor of poor prognosis in cervical cancer: a clinicopathologic study and a meta-analysis. Int J Gynecol Cancer (2014) 24:1054-64. doi: 10.1097/IGC.0000000000000162

27. Tang X, Zhang Q, Nishitani J, Brown J, Shi S, Le AD. Overexpression of human papillomavirus type 16 oncoproteins enhances hypoxia-inducible factor 1 alpha protein accumulation and vascular endothelial growth factor expression in human cervical carcinoma cells. Clin Cancer Res. (2007) 13:2568-76. doi: 10.1158/1078-0432.CCR-06-2704

28. Crawford RA, Caldwell C, Iles RK, Lowe D, Shepherd JH, Chard T. Prognostic significance of the bcl-2 apoptotic family of proteins in primary and recurrent cervical cancer. Br J Cancer (1998) 78:210-4.
29. Ferrandina G, Lauriola L, Distefano MG, Zannoni GF, Gessi M, Legge F, et al. Increased cyclooxygenase- 2 expression is associated with chemotherapy resistance and poor survival in cervical cancer patients. J Clin Oncol. (2002) 20:973-81. doi: 10.1200/JCO.2002.20.4.973

30. Soonthornthum T, Arias-Pulido H, Joste N, Lomo L, Muller C, Rutledge T, et al. Epidermal growth factor receptor as a biomarker for cervical cancer. Ann Oncol. (2011) 22:2166-78. doi: 10.1093/annonc/mdq723

31. Han R, Pacifici M, Iwamoto M, Trojanowska M. Endothelial Erg expression is required for embryogenesis and vascular integrity. Organogenesis (2015) 11:75-86. doi: 10.1080/15476278.2015.1031435

32. Nikolova-Krstevski V, Yuan L, Le Bras A, Vijayaraj P, Kondo M, Gebauer I, et al. ERG is required for the differentiation of embryonic stem cells along the endothelial lineage. BMC Dev Biol. (2009) 9:72. doi: 10.1186/1471213X-9-72

33. Pigazzi M, Masetti R, Martinolli F, Manara E, Beghin A, Rondelli R, et al. Presence of high-ERG expression is an independent unfavorable prognostic marker in MLL-rearranged childhood myeloid leukemia. Blood (2012) 119:1086-7; author reply 1087-8. doi: 10.1182/blood-2011-10-3 85815

34. Kron KJ, Murison A, Zhou S, Huang V, Yamaguchi TN, Shiah YJ, et al. TMPRSS2-ERG fusion co-opts master transcription factors and activates NOTCH signaling in primary prostate cancer. Nat Genet. (2017) 49:1336-45. doi: $10.1038 /$ ng.3930

35. Adamo P, Ladomery MR. The oncogene ERG: a key factor in prostate cancer. Oncogene (2016) 35:403-14. doi: 10.1038/onc.2015.109

36. Gupta S, Iljin K, Sara H, Mpindi JP, Mirtti T, Vainio P, et al. FZD4 as a mediator of ERG oncogene-induced WNT signaling and epithelial-to-mesenchymal transition in human prostate cancer cells. Cancer Res. (2010) 70:6735-45. doi: 10.1158/0008-5472.CAN-10-0244

37. Haber MA, Iranmahboob A, Thomas C, Liu M, Najjar A, Zagzag D. ERG is a novel and reliable marker for endothelial cells in central nervous system tumors. Clin Neuropathol. (2015) 34:117-27. doi: 10.5414/NP 300817

38. Giró-Perafita A, Sarrats A, Pérez-Bueno F, Oliveras G, Buxó M, Brunet J, et al. Fatty acid synthase expression and its association with clinicohistopathological features in triple-negative breast cancer. Oncotarget (2017) 8:74391-405. doi: 10.18632/oncotarget.20152

39. Van de Sande T, Roskams T, Lerut E, Joniau S, Van Poppel H, Verhoeven G, et al. High-level expression of fatty acid synthase in human prostate cancer tissues is linked to activation and nuclear localization of Akt/PKB.J Pathol. (2005) 206:214-9. doi: 10.1002/path.1760

40. Zaytseva YY, Harris JW, Mitov MI, Kim JT, Butterfield DA, Lee EY, et al. Increased expression of fatty acid synthase provides a survival advantage to colorectal cancer cells via upregulation of cellular respiration. Oncotarget (2015) 6:18891-904. doi: 10.18632/oncotarget.3783

41. Duan J, Sun L, Huang H, Wu Z, Wang L, Liao W. Overexpression of fatty acid synthase predicts a poor prognosis for human gastric cancer. Mol Med Rep. (2016) 13:3027-35. doi: 10.3892/mmr.2016.4902

42. Orita H, Coulter J, Lemmon C, Tully E, Vadlamudi A, Medghalchi SM, et al. Selective inhibition of fatty acid synthase for lung cancer treatment. Clin Cancer Res. (2007) 13:7139-45. doi: 10.1158/1078-0432.CCR-07-1186

43. Rahman MT, Nakayama K, Ishikawa M, Rahman M, Katagiri H, Katagiri A, et al. Fatty acid synthase is a potential therapeutic target in estrogen receptor/progesterone receptor-positive endometrioid endometrial cancer. Oncology (2013) 84:166-73. doi: 10.1159/000342967

Conflict of Interest Statement: The authors declare that the research was conducted in the absence of any commercial or financial relationships that could be construed as a potential conflict of interest.

Copyright (๑) 2018 Xia, Wang, Cai, Su, Shen, Meng, Chen, Li, Yan, Zhang and Xu. This is an open-access article distributed under the terms of the Creative Commons Attribution License (CC BY). The use, distribution or reproduction in other forums is permitted, provided the original author(s) and the copyright owner(s) are credited and that the original publication in this journal is cited, in accordance with accepted academic practice. No use, distribution or reproduction is permitted which does not comply with these terms. 